\title{
Performance Analysis of DSTBC over VHF Air-to-Ground Communication Channels
}

\author{
Amr A. Nagy \\ Military Technical College \\ Cairo, Egypt
}

\author{
Gamal M. Abdel-Hamid \\ Military Technical College \\ Cairo, Egypt
}

\author{
Ahmed E. Abdalla \\ Military Technical College \\ Cairo, Egypt
}

\begin{abstract}
This paper is concerned with evaluating the performance of differential space time block coding (DSTBC) over very high frequency (VHF) aeronautical communication channels (i.e. air-to-ground). Differential phase shift key (DPSK) is one of the differential transmission schemes that transmit information without full channel state information (CSI) at the receiver. There are two main problems that degrade the performance of the communication over this channel, multipath fading which is a common problem in any wireless channel and Doppler shifts which result from aircraft speeds. In order to combat such fading and improve the bit error rate (BER) performance of the communication system over this channel, the paper investigated the usage of multiple input multiple output (MIMO) systems with differential transmission scheme. This was done through computer simulations that analyze the performance of DSTBC using two antennas at the transmitter and $\mathrm{N}$ antennas at the receiver.
\end{abstract}

\section{General Terms}

Wireless communication, multi antenna system.

\section{Keywords}

Aeronauticalcommunication channels, DPSK, BER, MIMO, DSTBC

\section{INTRODUCTION}

Air-to-ground communications were defined in this paper as the communications between the aircraft and the ground radio station. There are many civilian and military applications which are based on these communications such as air traffic control (ATC) which is used for achieving a high efficient and safe air flights [1], navigation systems such as distance measuring equipment (DME) which supply the aircraft with its distance from the ground station and automatic dependent surveillance broadcast (ADS-B)which is a surveillance technology for tracking aircrafts. There are specified frequency bands for every application. This paper was concerned with the aeronautical communications over the VHF band. The VHF digital link (VDL) (118 MHz to 137 $\mathrm{MHz}$ ) with $25 \mathrm{kHz}$ channel spacingis a communication standard was divided into 760 channels and was assigned for both civilian and military air to ground communications [1, 2]. A stochastic model for the air to ground channel was proposed by Haas [3, 4]. This model describes the propagation between aircraft and ground terminal as multipath propagation which is a common problem in wireless communications as shown in figure 1. Multipath propagations occur when there exist multiple propagation paths between the transmitter and receiver. These multiple propagation paths result from reflections and scattering of the propagating electromagnetic field by objects (mountains, Buildings, etc.) in the propagation environment. The degrees to which these reflections distort the received signal depend on the number, the strength of the reflections which in turn is a function of the electric properties of the reflectors and scatterers, and the discrimination of the antenna gain pattern in the direction of the arriving reflections [5].

The aeronautical channel model divided the flight into four different scenarios: En-route, Arrival, Taxi and Parking. Each of these scenarios has its own parameters which is characterizing the type of fading, Doppler, and delay. By comparing aeronautical channel with the traditional terrestrial mobile radio channels, air-to-ground channels not only suffer from multipath fading which is less effective on angle modulation signals than linear modulation signals but also suffer from large Doppler spread that can complicate the problem of estimating the channel accurately for coherent detection. For such channel ( high-mobility environment) it may be difficult or costly to estimate the channel accurately [6], so received signals can be detected non-coherently using DPSK which can be demodulated without the use of channel estimates. Appropriate modulation schemes should be used, e.g., differential quadrature phase shift keying (DQPSK) used in military Link-11 and differential 8 phase shift keying (D8PSK) used in VDL mode 2 [2]. Due to the poor BER performance of this communication system, there was necessary need to mitigate fading effects. This paper investigated the benefits of using space time coding to combat such fading.

The BER performance of different phase shift keying modulation schemes in the aeronautical channel was analyzed in [2]which compared between themand the performance of different coherent modulation schemes. The differential modulation schemes are more suitable for aeronautical channels due to its ability to reduce the complexity of the receiver and withstand the Doppler shift [2]. The author in references $[3,7]$ utilized the frequency diversity to improve the performance of the aeronautical communications in the $\mathrm{C}$ band. The remainder of the paper is structured as follows: section II presents the aeronautical channel model. The different benefits of MIMO for wireless communications are introduced in section III. Unitary DSTBC BER performance analysis over air-to-ground communication channels were presented in section IV. Finally, section V concludesthe paper. 


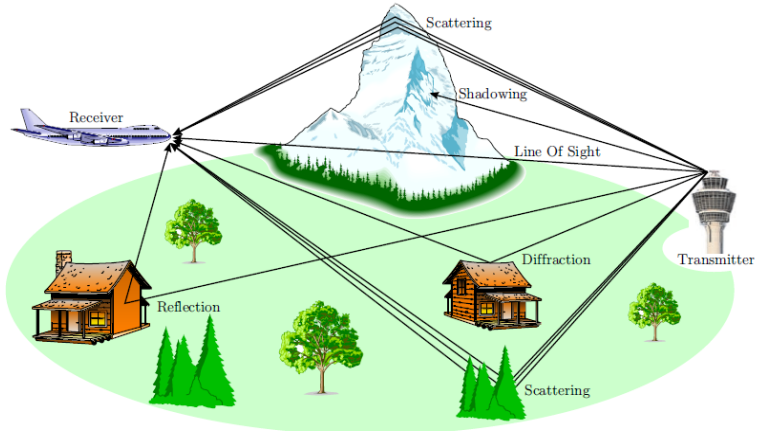

Fig 1: Multipath propagation in air-to-ground communications systems[8]

\section{AERONAUTICAL CHANNEL}

\section{MODEL}

The aircraft during the flight subjected to different conditions leads to different channel scenarios. These scenarios are characterized by the type of fading, the Doppler, and the delays in the system. The flight divided into four scenarios $[3,4]$ : En-route scenario, Arrival and takeoff scenario, Taxi scenario, and Parking scenario.

\subsection{En-route scenario}

The en-route scenario is applied when the aircraft is airborne and communicates with the ground site as shown in figure 2 .

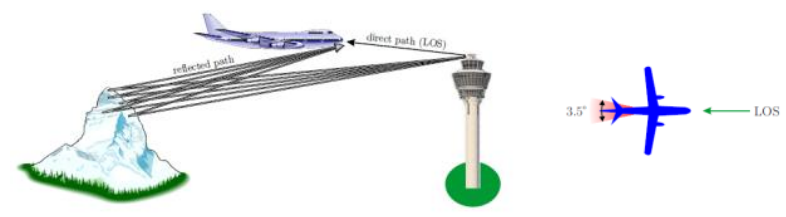

Fig. 2: Multipath propagation for en-route scenario[8]

As shown in figure 2 the aircraft receives multipath signals consisting of a LOS path and a cluster of reflected, delayed paths. Therefore, this scenario may be characterized by Rician fading with rice factor $\mathrm{k}=[2-20] \mathrm{dB}[3]$.

$$
\mathrm{K}=\frac{\mathrm{a}^{2}}{\mathrm{c}^{2}}
$$

Where $\mathrm{K}$ is the relation between the power of the LOS component $\mathrm{a}^{2}$ and the average power of the scattered component $\mathrm{C}^{2}[3]$. When $\mathrm{K}$ is large (greater than $20 \mathrm{~dB}$ ), the channel is well approximated by an Additive White Gaussian Noise (AWGN) channel and when $\mathrm{K}$ is small (smaller than $20 \mathrm{~dB}$ ) the channel is well approximated by the Rayleigh fading channel [5]. The speed of the aircraft takes value from the range $17: 440 \mathrm{~m} / \mathrm{s}$ so this scenario is characterized by fast fading due to the resulting Doppler spread.

$$
f_{d}=\frac{v}{\lambda} \cos \theta
$$

Where $v$ is the relative speed between the transmitter and receiver, $\lambda$ is the carrier wave length and $\theta$ is the angle of arrival (AOA) of the received signal.

As shown in figure 2 assuming the direction of the LOS path coincides with the heading of the aircraft resulting in a carrier shift of the LOS path by $f_{\text {Dmax }}$ (maximum Doppler spread), whereas the scattered components come from behind. The scatterers assumed to be uniformly distributed within beamwidth of diffuses component with $\beta=3.5^{\circ}$, i.e. the angles of arrival have a small range $[\pi-\beta / 2, \pi+\beta / 2]$ that's due to high altitude (assume $10 \mathrm{Km}$ ) of the aircraft. The Doppler spread $f_{d}$ is a random variable with probability density function (pdf) known as Jakes distribution with maximum value $=200 \mathrm{~Hz}$ at $137 \mathrm{MHz}$ carrier frequency fc [4].

$p_{f_{D}}\left(f_{D}\right)=\left\{\begin{array}{cl}\frac{1}{\pi f_{D_{\max }} \sqrt{1-\left(\frac{f_{D}}{f_{D_{\max }}}\right)^{2}}}, \text { if }\left|f_{D}\right|<f_{D_{\max }} \\ 0, \quad \text { else }\end{array}\right.$

The maximum delay $\tau_{\max }=33 \mu \mathrm{s}, \tau=\Delta \mathrm{d} / \mathrm{c}$ and $\Delta \mathrm{d} \approx \mathrm{h}$ (aircraft altitude) [3].

\subsection{Arrival and takeoff scenario}

The arrival and takeoff scenario is applied when the aircraft is about to land (or increase its speed and altitude to go en-route) and communicates with the ground site as shown in shown figure 3 .
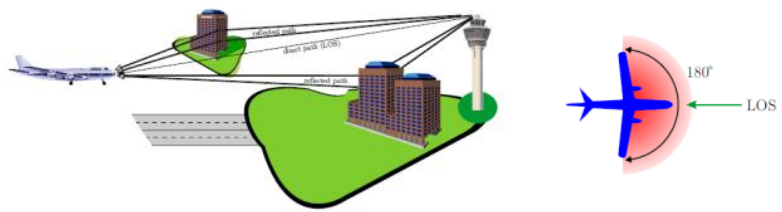

\section{Fig. 3: Multipath propagation for arrival scenario[8]}

It can be assumed that the LOS path is present during this scenario while the aircraft is still airborne. On the other hand, also there will be scattered path components, mainly from buildings at the airport itself. The result is again a Rician channel krice $=[9-20] \mathrm{dB}[3,4]$. The aircraft speed during the arrivalassumed to be $\mathrm{V}=25: 150 \mathrm{~m} / \mathrm{s}$ so this scenario is characterized by Rician fast fading. The beam width of scattered components is $\beta=180^{\circ}$; the scattered components is broader than in the en-route environment. Since the aircraft is still some distance away from the airport and maximum delay $\tau_{\max } \approx 7 \mu \mathrm{s}[3,4]$.

\subsection{Taxi scenario}

The taxi scenario is applied when the aircraft is on the ground and travelling toward or from the terminal, as shown in figure 4

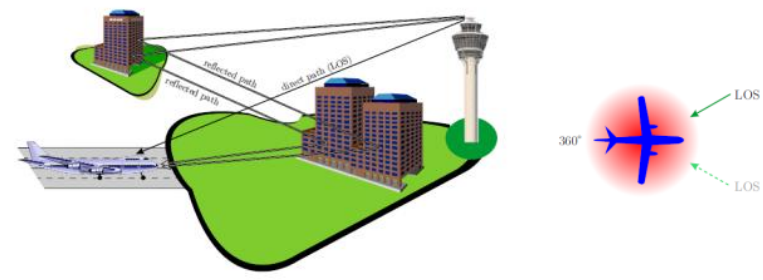

Fig. 4: Multipath propagation for taxi scenario[8]

This scenario is characterized by Rician fading. The aircraft speeds $V=0 \ldots 15 \mathrm{~m} / \mathrm{s}$ during taxi. The beam width of the scattered components is $\beta=360^{\circ}$ and Krice $\approx 6.9 \mathrm{~dB}$. The maximum excess delays $\tau_{\max } \approx 0.7 \mu \mathrm{s}[3,4]$. 


\subsection{Parking scenario}

The parking scenario is applied when the aircraft is on the ground and travelling at very slow speed close to the terminal or is parked at the terminal, as shown in figure 5.
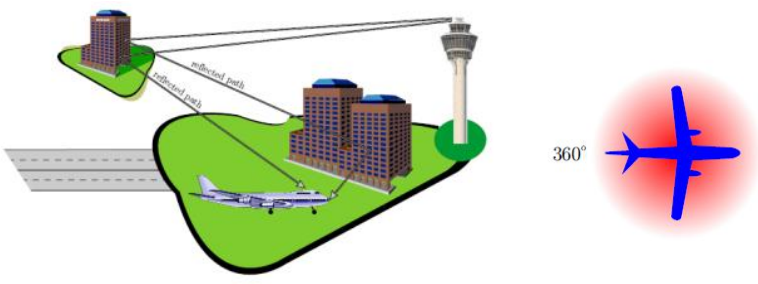

Fig.5: Multipath propagation for parking scenario[8]

The LOS path is assumed to be blocked in this scenario, which results in Rayleigh fading. Although the airport control tower should always be installed in a place where there is a line of sight to all aircrafts during taxiing or parking but due to the high density at airports, that is not always possible. Due to the fact that the aircraft is parked at the terminal or travelling at very slow speed, the fading is even slower than in the taxi scenario $\mathrm{V}=0 . .5 \mathrm{~m} / \mathrm{s}$, the beam width of the scattered components is $\beta=360^{\circ}$, Krice $=0 \mathrm{~dB}$. The maximum delay $\tau_{\max } \approx 0.7 \mu \mathrm{s}[3,4]$.

\section{BENEFITS OF MIMO FOR WIRELESS COMMUNICATIONS}

The benefits from multiple antennas techniques arise from the use of a new dimension-space. The spatial dimension comes as a complement to time and/or frequency dimensions [9]. There are three main benefits of using multiple antennas in wireless communication systems: a multiplexing gain, an antenna gain, or a diversity gain as shown in figure 6 .

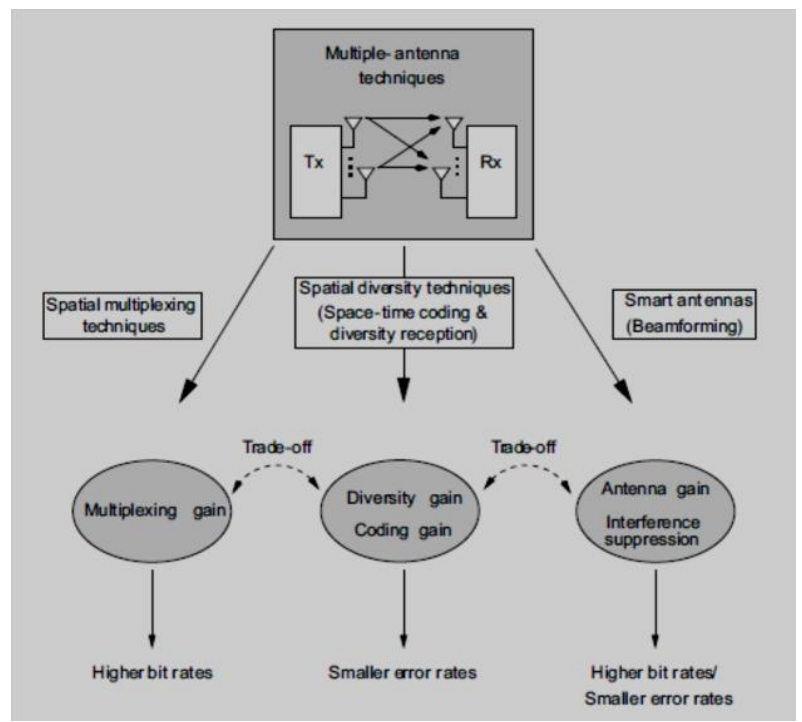

Fig. 6: Benefits of multiple-antenna techniques for wireless communications[9]

This paper is interested in improving the BER performance of the single input single output (SISO) air-to-ground communications so; it takes advantages of the diversity gain resulting from the use of multiple antennas techniques. The main idea of spatial diversity techniques is to provide a diversity and/or coding gain by sending redundant signals over multiple transmit antennas. Alamouti was the pioneer in this area of research. He proposed a simple technique for two transmit antennas [10] but this technique estimates CSI which may be difficult or costly in our case. DSTBC is another technique based on Alamouti technique.

This technique does not require estimating CSI because the encoding and decoding processes are done in differential way as explained in the next section.

\section{UNITARY DSTBC BER PERFORMANCE ANALYSIS}

\subsection{DSTBC process}

Tarokh and Jafarkhani presented a differential detection scheme for Alamouti's code[11]. The DSTBC scheme can be described as follows: Two information symbols $x_{1}$ and $x_{2}$ are buffered by the transmitter and transmitted in two time slots in the following manner. In the first time slot, symbol $\mathrm{x}_{1}$ is transmitted over the first antenna and simultaneously symbol $\mathrm{x}_{2}$ is transmitted over the second antenna. In the second time slot, signal $-\mathrm{X}_{2}$ is transmitted by the first antenna and $\mathrm{X}{ }_{1}$ is transmitted by the second antenna. In general, STBC scheme encodes $\mathrm{K}$ complex symbols $\mathrm{x}_{1}, \ldots, \mathrm{x}_{\mathrm{K}}$ by including linear combinations of $\pm x_{1}, \ldots \ldots, \pm x_{K}$ and their conjugates $\pm x_{1}^{*}, \ldots \ldots$, $\pm \mathrm{X}_{\mathrm{K}}$ in the code matrix. This can be expressed in a following form as:

$$
V=\frac{1}{\sqrt{2}} \sum_{i=1}^{2} A_{i} x_{i}+B_{i} x_{i}^{*}
$$

Where $A_{i}$ and $B_{i} \quad\{\mathrm{i}=1,2\}$ are known as dispersion matrices since they disperse the symbols over the transmit antennas. $\frac{1}{\sqrt{2}}$ is a normalization factor used to make the total transmitted power from the two antennas at one time slot to be one [6]. The dispersion matrices are:

$$
\begin{aligned}
& A_{1}=\left[\begin{array}{ll}
1 & 0 \\
0 & 0
\end{array}\right], \quad B_{1}=\left[\begin{array}{ll}
0 & 0 \\
0 & 1
\end{array}\right], \\
& A_{2}=\left[\begin{array}{ll}
0 & 1 \\
0 & 0
\end{array}\right], \quad B_{2}=\left[\begin{array}{cc}
0 & -1 \\
0 & 0
\end{array}\right]
\end{aligned}
$$

And the first code matrix can be written as:

$$
v_{1}=\frac{1}{\sqrt{2}}\left[\begin{array}{cc}
x_{1} & x_{2} \\
-x_{2}^{*} & x_{1}^{*}
\end{array}\right]
$$

Then these code matrices were differentially encoded to construct the transmitted matrices as follows:

$$
s_{t}=v_{t} s_{t-1}
$$

Where $s_{t}$ is the transmitted matrix at time $t, v_{t}$ is the code matrix at time t. After passing through the cannel the received matrices can be represented as:

$$
Y_{t}=H S_{t}+N_{t}
$$

Where $Y_{t}$ is the received matrix at time $t, H$ is $2 x N$ channel matrix $(\mathrm{N}=1,2,4)$ and $\mathrm{N}_{\mathrm{t}}$ is $2 \mathrm{xN}$ noise matrix representing separate AWGN to each path. Finally maximum likelihood (ML) differential decoding is given by [6]: 


$$
\hat{v}_{t}=\arg \max _{l \in Q}\left\|y_{t-1}+y_{t} v_{l}^{H}\right\|
$$

Where $\mathrm{Q}=\{1,2,3,4\},\|$.$\| denotes the frobenius norm of a$ matrix. The following block diagram represents the differential STBC scheme over $2 \mathrm{xN}$ communication system.

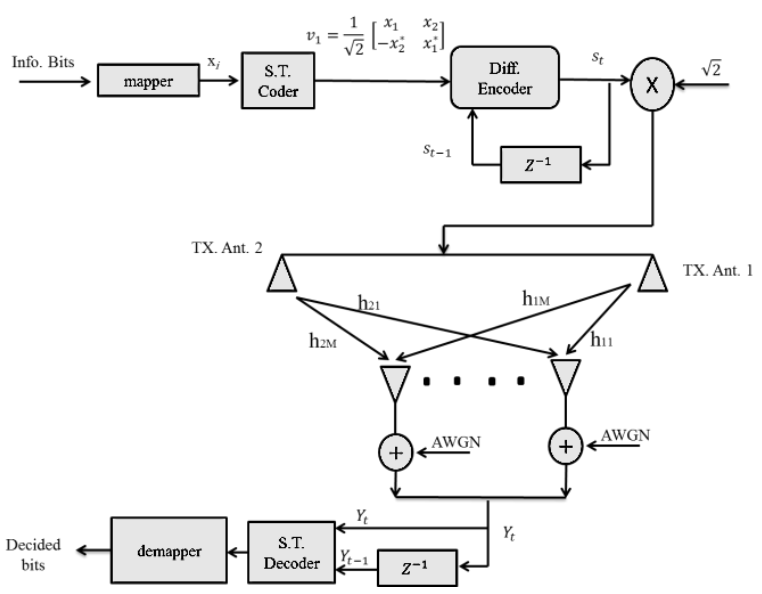

Fig. 7: block diagram showing the differential transmission of an STBC scheme over an 2XN communication system

As shown in the block diagram in figure 7 , the information bits were mapped by binary phase shift keying (BPSK) encoder. Then the mapped information symbols $x_{i}$ are encoded by DSTBC as explained in the previous section. The first column of the encoded matrix is transmitted through the first antenna and the second column is transmitted through the second antenna. This simulation used $137 \mathrm{MHz}$ as a carrierfrequency. The fading effect on every path between the different antennas assumed to have decorrelation as seen in figure 7. The DSTB decoding process was explained in the previous section.

\subsection{Simulation results}

Figures 8-12 represent the simulation results of using DSTBC withorder $2 \mathrm{xN}$ over the different scenarios of the aeronautical channel. These simulations were done by using MATLAB. The results of these simulation show that, the performance of the communication system utilizing DSTBC is better than the SISO system utilizing DPSK. It also shows that, the performance of DSTBC is enhanced by increasing the number of receiving antennas.

Figure 8 Shows the en_route scenario assuming Krice $=2 \mathrm{~dB}$, $f_{d} T_{b}=.02$. The results show that for the case, when $E_{b} / N_{o}=8$ $\mathrm{dB}, \mathrm{BER}(\mathrm{DBPSK})=0.0513$, BER $(2 \mathrm{x} 1)=0.0352$, BER $(2 \times 2)$ $=4.38 \mathrm{e}-3$, BER $(2 \times 4)=1.10 \mathrm{e}-4$, i.e., by increasing the number of antennas at the receiver the BER improved. Figure 9 is representing the en_route scenario assuming Krice $=10 \mathrm{~dB}$, $\mathrm{f}_{\mathrm{d}} \mathrm{T}_{\mathrm{b}}=.02$, when $\mathrm{E}_{\mathrm{b}} / \mathrm{N}_{\mathrm{o}}=8 \mathrm{~dB}$, BER $($ DBPSK) $=0.01212$, BER $(2 \times 1)=0.0352$, BER $(2 \times 2)=9.66 e-3$, BER $(2 \times 4)=2.66 e-6$. Figure 10 is representing the arrival scenario assuming Krice $=9 \mathrm{~dB}, \mathrm{f}_{\mathrm{d}} \mathrm{T}_{\mathrm{b}}=0.0068$, when $\mathrm{E}_{\mathrm{b}} / \mathrm{N}_{\mathrm{o}}=8 \mathrm{~dB}, \mathrm{BER}$ (DBPSK) $=0.011$, BER $(2 \times 1)=0.011, \operatorname{BER}(2 \times 2)=5.04 \mathrm{e}-4$, BER $(2 \times 4)$ $=2.67 \mathrm{e}-6$. Figure 11 is representing the performance of both
DSTBC $2 \mathrm{XN}$ and DBPSK in taxi scenario assuming Krice $=6.9 \mathrm{~dB}, \mathrm{f}_{\mathrm{d}} \mathrm{T}_{\mathrm{b}}=0.0006$, when $\mathrm{E}_{\mathrm{b}} / \mathrm{N}_{\mathrm{o}}=8 \mathrm{~dB}$, BER $($ DBPSK $)=1.95 \mathrm{e}-2$, BER $(2 \times 1)=1.33 \mathrm{e}-2$, BER $(2 \times 2)=7.65 \mathrm{e}-$ 4 , BER $(2 \times 4)=1.33 e-6$. Figure 12 is representing the performance of both DSTBC $2 \mathrm{XN}$ and DBPSK in parking scenario assuming Krice $=0 \mathrm{~dB}, \mathrm{f}_{\mathrm{d}} \mathrm{T}_{\mathrm{b}}=0.0002$, when $\mathrm{E}_{\mathrm{b}} / \mathrm{N}_{\mathrm{o}}=8$ $\mathrm{dB}$, BER $($ DBPSK $)=6.84 \mathrm{e}-2$, BER $(2 \times 1)=3.36 \mathrm{e}-2$, BER $(2 \times 2)=5.36 \mathrm{e}-3$, BER $(2 \times 4)=6.66 \mathrm{e}-5$

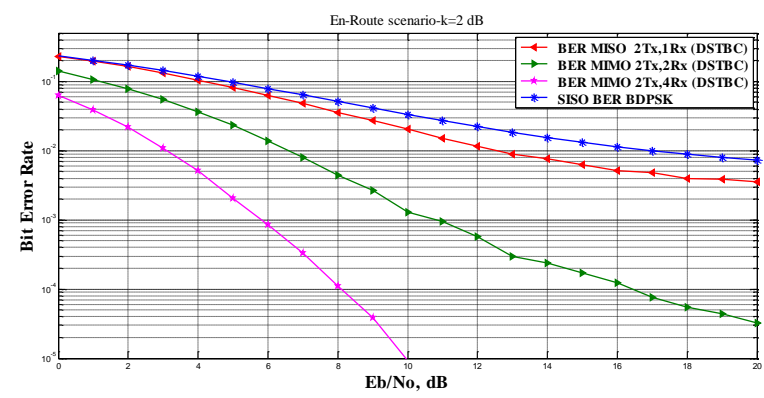

Fig. 8: Performance of DSTBC 2XN over the aeronautical communication channel (En-route scenario) Krice $=2 \mathrm{~dB}$

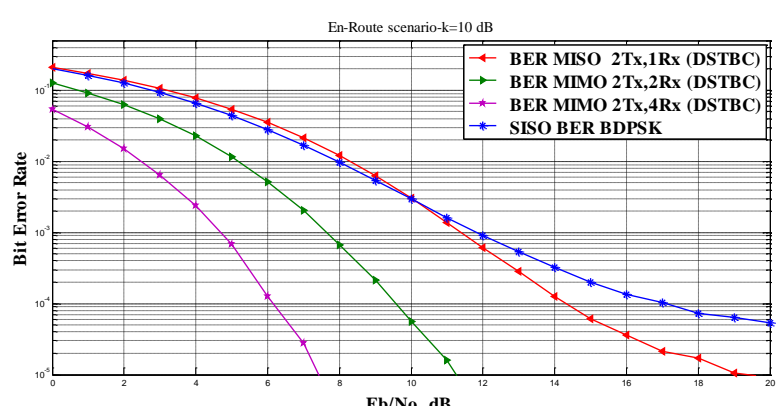

Fig. 9: Performance of DSTBC 2XN over the aeronautical communication channel (En-route scenario) Krice $=10 \mathrm{~dB}$

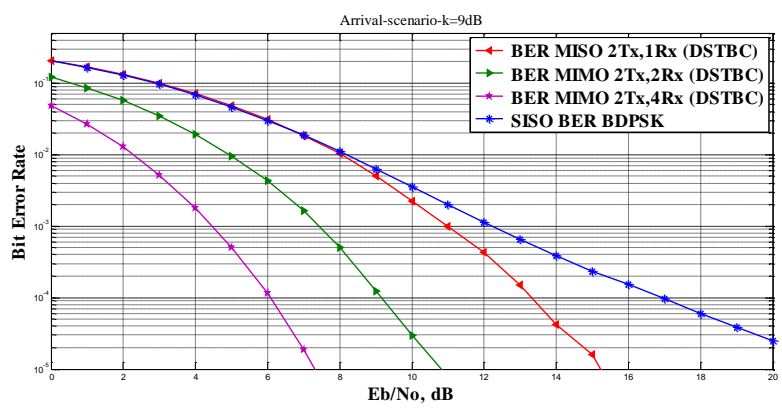

Fig. 10: Performance of DSTBC 2XN over the aeronautical communication channel (Arrival scenario)

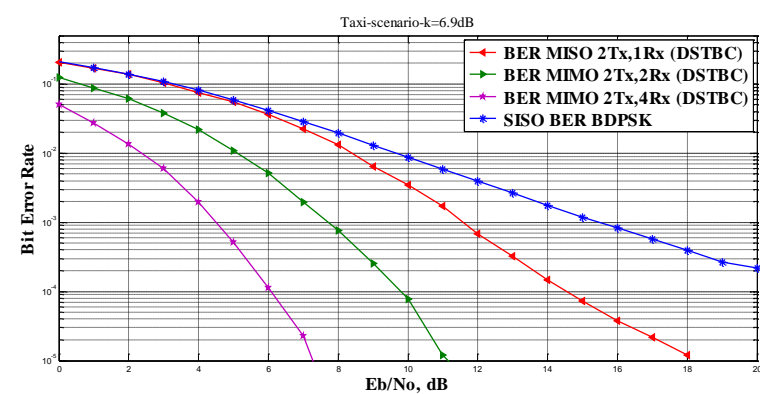

Fig. 11: performance of DSTBC 2XN over the aeronautical communication channel (Taxi scenario) 


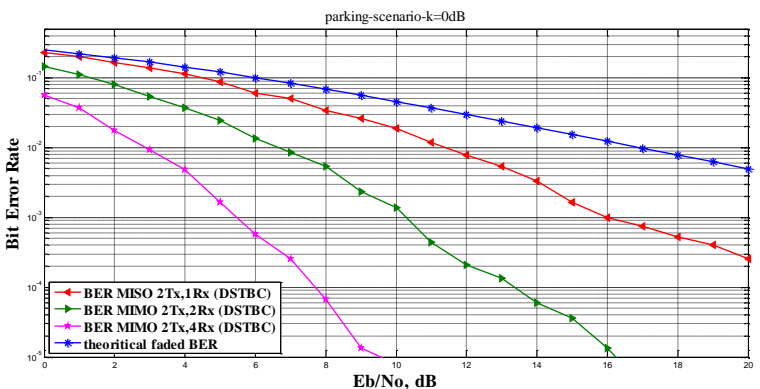

Fig. 12: performance of DSTBC 2XN over the aeronautical communication channel (Parking scenario)

\section{CONCLUSIONS}

This paper discussed the different scenarios of the aircraft flight (En-route, Arrival, Taxi, and Parking); the behavior of the air-to-ground communication channel was introduced at each of these scenarios. The BER results of the SISO show that multipath fading and Doppler shifts in the air-to-ground communication channel degrade the probability of error. In order to combat fading and improve the BER performance of air-to-ground communications, the paper investigated the usage of MIMO communication systems. This was done through computer simulations that analyze the performance of DSTBC using two antennas at the transmitter and $\mathrm{N}$ antennas at the receiver. The results show that the performances were enhanced due to using DSTBC.

\section{REFERENCES}

[1] S. M. Elnoubi, "A simplified stochastic model for the aeronautical mobile radio channel," 1992, pp. 960-963 vol. 2.

[2] M. Gong, C. Zhang, H. Han, and X.Lin "Performance Analysis of Different Phase Shift Keying Modulation Schemes in Aeronautical Channels*," Tsinghua Science \& Technology, vol. 14, pp. 1-6, 2009.
[3] E. Haas, "Aeronautical channel modeling," Vehicular Technology, IEEE Transactions on, vol. 51, pp. 254-264, 2002.

[4] P. Hoeher and E. Haas, "Aeronautical channel modeling at VHF-band," 1999, pp. 1961-1966 vol. 4.

[5] M. Rice, R.Dye, and K.Welling, "Narrowband channel model for aeronautical telemetry," Aerospace and Electronic Systems, IEEE Transactions on, vol. 36, pp. 1371-1376, 2000.

[6] B. Vucetic and J. Yuan., Space-Time Coding. England: John Wiley \& Sons Ltd, 2003.

[7] E. Haas, et al., "Development and implementation of an advanced airport data link based on multi-carrier communications," European transactions on telecommunications, vol. 13, pp. 447-454, 2002.

[8] E. Haas, "Design, evaluation and implementation of a multi-carrier transmission system for aeronautical communications," phd theis, Munich,Germany, Oct.2002.

[9] R. J. S. Mietzner, L.Lampe, W.H.Gerstacker, and P.A.Hoeher, "Multiple-antenna techniques for wireless communications-a comprehensive literature survey," Communications Surveys \& Tutorials, IEEE, vol. 11, pp. 87-105, 2009.

[10] S. M. Alamouti, "A simple transmit diversity technique for wireless communications," Selected Areas in Communications, IEEE Journal on, vol. 16, pp. 14511458,1998

[11] V. Tarokh and H. Jafarkhani, "A differential detection scheme for transmit diversity," Selected Areas in Communications, IEEE Journal on, vol. 18, pp. 11691174, 2000. 